\title{
Die Katalogisierung der Handschriften in der WLB
}

\section{Die Anfänge}

Im Jahr 1765 gründete Herzog Carl Eugen die „Herzogliche Öffentliche Bibliothek", die von Anfang an auch eine beträchtliche Zahl von Handschriften enthielt. Allerdings handelte es sich dabei überwiegend um Werke, die erst in der Neuzeit entstanden waren. Diese dienten zum einen praktischen Zwecken der Landesregierung wie Rechnungsbücher, Militaria oder Kartenwerke, zum anderen waren es Chroniken und historische Werke, die seit jeher zu den besonderen Interessensgebieten von Fürsten gehörten. Darüber hinaus gab es große Bestände an Musikalien, die vor allem Materialien zu den Aufführungen am Hoftheater überliefern, sowie Zeugnisse von festlichen Ereignissen wie dem Stuttgarter Armbrustschießen von 1560 (Cod. hist. $2^{\circ} 165$ ). Vergleichsweise wenige Codices stammen noch aus dem - späten - Mittelalter, im Wesentlichen solche, die im privaten Auftrag der württembergischen Regenten oder anderer Mitglieder der Herrscherfamilie entstanden wie das Gebetbuch Eberhards im Bart (Cod. brev. 1), oder auf anderem Wege an das Haus Württemberg gelangt waren wie die BoccaccioHandschrift (Cod. hist. $2^{\circ} 13$ ), die möglicherweise von Mechthild von der Pfalz, der Mutter Eberhards, mitgebracht wurde. Bedeutenden Zuwachs hatte die Bibliothek auch durch Käufe des Gründers und großen Büchersammlers Carl Eugen in ganz Europa erfahren. Zu einem Teil ihres Grundstocks wurde die Sammlung des Hofbibliothekars und Professors an der Kunstakademie Joseph Uriot' ${ }^{1}$, die der Herzog diesem 1764 abkaufte; die Handschriften und Inkunabeln aus seinem Besitz kann man heute noch an ihren Einbandrücken erkennen. Erst mit der Säkularisation kam mit den Bibliotheken der württembergischen Klöster der weitaus größte Teil des heutigen Bestands an mittelalterlichen Handschriften hinzu.

Nach der Gründung vermehrte Carl Eugen die Bestände, auch der Handschriften, weiterhin durch die Zukäufe, so zum Beispiel im Jahr 1785 mit der Sammlung des Konsistoriumsdirektors Friedrich Wilhelm Frommann, über die der ursprünglich auch für Eberhard im Bart geschriebene Columella-Codex
(Cod. cam. et oec. $\left.2^{\circ} 1\right)^{2}$ wieder in den Besitz des Hauses Württemberg zurückgelangte. Wie in jeder größeren Bibliothek bedurfte es einer Ordnung. Man teilte die Bestände, wie einem Bericht von 1781 zu entnehmen ist, nach Fächern in Gruppen ein: Gottesgelehrsamkeit, Rechtswissenschaften, Historische Wissenschaften und weitere Gruppen, an achter und letzter Stelle Manuscripta ${ }^{3}$. Die dringendste Aufgabe war es, Kataloge anzulegen, darunter einen speziellen für die Handschriften. Carl Eugen befahl 1788 deren Katalogisierung; es ist jedoch ungewiss, ob gleich damit begonnen wurde. Diese Aufgabe übernahm der Oberbibliothekar Johann Gottlieb Schott ${ }^{4}$, der den Bestand in 13 Gruppen einteilte mit entsprechender Signaturvergabe (Codices biblici,

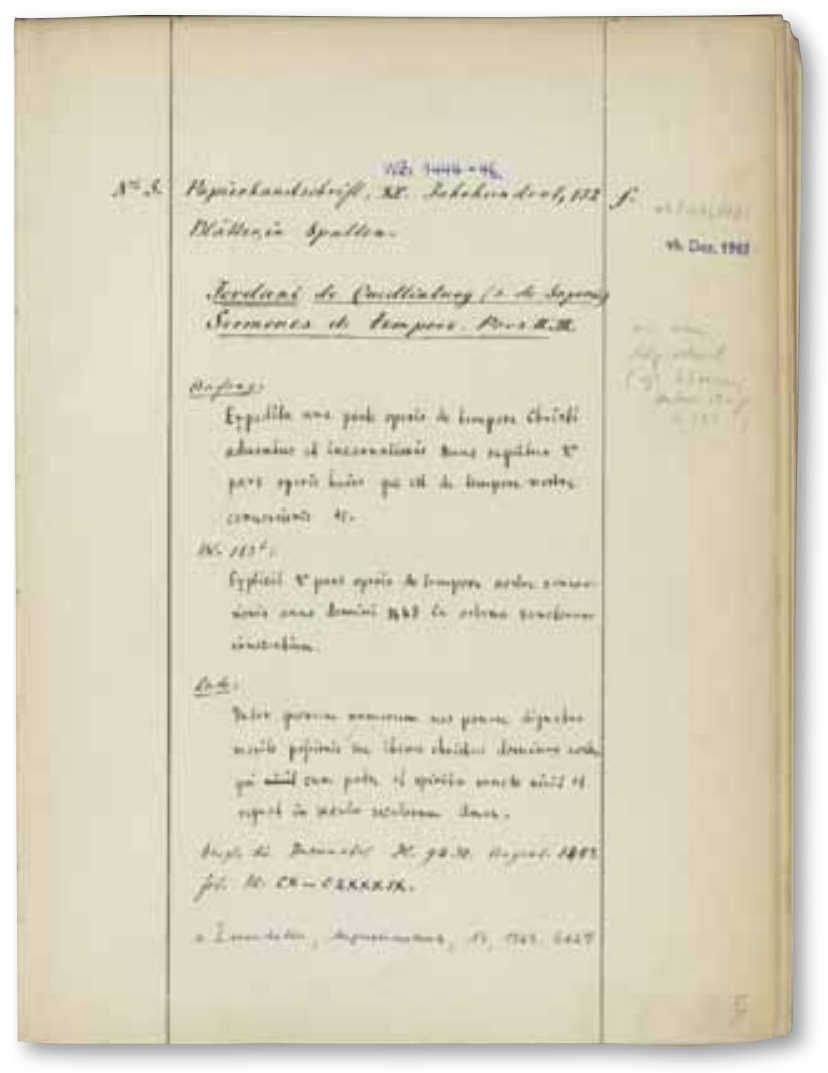

Abb. 1: Bandkatalog zu Cod.theol. et phil.fol. 1-150

(1) Karl Löffler, Geschichte der Württembergischen Landesbibliothek, Leipzig 1923 (ZfB, Beiheft 50), Nachdr. Nendeln 1968, S. 246. Dazu auch: Kerstin Losert, in: 250 Jahre Württembergische Landesbibliothek (Ausst.-Kat.), Stuttgart 2015, S. 33.

(2) Karl Löffler, L. Junius Moderatus Columella, De re rustica, übers. durch Heinrich Oesterreicher, Abt von Schussenried. 2 Bde. (Bibliothek des Litterarischen Vereins in Stuttgart 263), Tübingen 1914, Bd. 1, S. XIV.

(3) Löfler, wie Anm. 1, S. 45.

(4) Löffler, wie Anm. 1, S. 83. Dazu auch: Kerstin Losert, wie Anm. 1, S. 96-101 
Cod. breviarii etc.), die so noch heute besteht, allerdings nicht in ihrer ursprünglichen Reihenfolge. Schott widmete sich bis an sein Lebensende 1813 dieser Aufgabe und initiierte einen Katalog in 13 großen Bänden, in den er auch die durch die Säkularisation neu hinzugekommenen Codices einarbeitete. Wesentlichen Anteil daran hat aber Franz Pfeiffer ${ }^{5}$, der ab 1847 die meisten Katalogbeschreibungen verfasste und teilweise überarbeitete. Diese Bände sind bis heute in Benutzung geblieben, soweit nicht moderne Kataloge zur Verfügung stehen wie zu den Codices breviarii und den Codices poetici.

Über 100 Jahre nach Gründung der Bibliothek gab das 25. Thronjubiläum König Karls im Jahre 1889 den Anlass für das Projekt eines ersten gedruckten Teilkatalogs der Handschriftensammlung, den man dem Jubilar als Geschenk präsentieren wollte. Der Bibliothekar Wilhelm von Heyd (unter Mithilfe anderer Bibliothekare) hatte die Codices historici schon so weit bearbeitet, dass ein erster Teil praktisch druckfertig vorlag, und so konnte dieser 1891 in zwei Bänden erscheinen ${ }^{6}$; der zweite Teil ist nie erschienen. Zur gleichen Zeit plante der Justizreferendar Emil Seckel einen gedruckten Katalog der juristischen Handschriften, der aber unter anderem aus systematischen Gründen nicht zu dem Katalog von Heyds passte; Seckel hatte juristische Texte aus der Hofbibliothek (HB VI ... und andere) mit aufgenommen. Sein handschriftlicher Katalog liegt aber als (foliierter!) Codex in Buchform vor und kann in der Handschriftenabteilung benutzt werden? . Beide Werke bieten, gemessen an modernen Katalogen, äußerst knappe Informationen, die sich meist auf die Benennung der Texte beschränken.

\section{Das große Projekt der wissenschaft- lichen Erschließung}

In den folgenden sehr schwierigen Jahrzehnten, die durch die beiden Weltkriege geprägt waren, gab es in Stuttgart keine nennenswerten Bemühungen auf diesem Gebiet. 1945 war Wilhelm Hoffmann

(5) Löffler, wie Anm. 1, S. 250.

(6) Wilhelm von Heyd, Die historischen Handschriften der Königlichen öffentlichen Bibliothek zu Stuttgart, 2 Bde, Stuttgart 1889-1891. Vgl. auch Löffler, wie Anm. 1, S. 230 und 251.

(7) Emil Seckel, Verzeichnis der mittelalterlichen Rechtshandschriften der königlichen Bibliothek zu Stuttgart (Cod. hist. $4^{\circ}$ 522), vermutlich 1889.

(8) Dazu auch Alessandra Sorbello Staub, in: Das Mittelalter. Perspektiven mediävistischer Forschung. Zeitschrift des Mediävistenverbandes 14/2009, Heft 2, S. 14

(9) Johanne Autenrieth, Die Handschriften der Württembergischen Landesbibliothek Stuttgart 2.3: HB VI 1-VII 71, Wiesbaden 1963.

(10) Derzeit gültig: Deutsche Forschungsgemeinschaft, Richtlinien Handschriftenkatalogisierung, 5. Aufl., Bonn-Bad Godesberg 1992.
Direktor der Landesbibliothek (bis 1970) geworden. Er hatte seit 1938 selbst die Handschriftenabteilung geleitet, engagierte sich sehr früh für die Erschließung der Handschriftenbestände in Stuttgart und war 1960 maßgeblich beteiligt an dem entscheidenden Anstoß, nämlich dem Beschluss der Deutschen Forschungsgemeinschaft, ein großes Projekt zur Katalogisierung sämtlicher abendländischer Handschriften in den Bibliotheken und einigen anderen Institutionen Deutschlands zu initiieren und zu finanzieren $^{8}$. In Stuttgart hatte Johanne Autenrieth bereits 1957 begonnen mit einer Beschreibung der Signaturengruppen HB VI und HB VII (Codices juridici et politici; Patres) der Hofbibliothek, für die ja bis dahin überhaupt keine gedruckten Kataloge vorlagen. Im Jahre 1963 erschien der gedruckte Katalog ${ }^{9}$, der die Beschreibungen in einer Form bot, die mit zur Grundlage der - später noch einige Male überarbeiteten - Richtlinien der DFG ${ }^{10}$ wurde. Diese für die Bearbeiter verbindliche Anlage der Katalogisate sieht in vereinfachter Darstellung aus wie folgt:

- Signatur.

- Überschrift: Autoren oder Sachtitel.

- Schlagzeile: Beschreibstoff - Blattzahl - Maße Entstehungsort oder -region und Datierung.

- Kodikologie: Wasserzeichen. Lagen. Schriftraum, Spaltenzahl, Zeilenzahl. Schriftart. Auszeichnungsschriften. Buchschmuck.

- Einband (Material, Stempel). Fragmente im Einband.

- Geschichte der Handschrift: Schreiber, frühere Besitzer.

- Literatur zur Handschrift.

- Texte: Folioangabe, Autor, Titel; Incipit, Explicit. Literatur (Editionen, Repertorien).

Dieses immer gleiche Schema ermöglicht es dem Benutzer, sehr schnell Antworten auf seine Fragen zur Handschrift zu finden, sei es zum Äußeren oder zu den Texten. Wer ältere Handschriftenkataloge kennt, sieht, dass den materiellen und historischen Aspekten jetzt mehr Gewicht verliehen wird, entsprechend den erweiterten Fragestellungen der Benutzer, die sich nicht mehr nur auf die Texte beziehen. Zusätzlich enthalten die Kataloge außer einem Literaturverzeichnis ein Orts-, Sach- und Personenregister sowie ein Register der Textanfänge. Diese Register wurden aus allen Katalogen, die im DFG-Programm entstanden waren, fortlaufend zum sogenannten Gesamtindex zusammengeführt, der 
anfangs periodisch als jeweils erweiterte MicroficheEdition erschien, später über Internet zugänglich war, so dass bei der Recherche nicht jedes Mal die Register aller einzelnen Kataloge aufgeschlagen werden mussten. Mittlerweile sind die meisten bisher erschienenen Kataloge in der Datenbank Manuscripta mediaevalia erfasst, die eine Suche unter allen relevanten Aspekten ermöglicht und zusätzlich die Katalogseiten als Scans bietet ${ }^{11}$.

Um die Qualität der Arbeiten zu gewährleisten, wurden fünf Bibliotheken, die bedeutende Handschriftensammlungen mit kompetenter Leitung besaßen und die nötigen Arbeitsmittel (Fachliteratur, Repertorien etc.) zur Verfügung stellen konnten, als sogenannte Handschriftenzentren eingesetzt, die neben den eigenen auch die Bestände anderer Bibliotheken bearbeiten sollten. Neben Berlin, Wolfenbüttel, Frankfurt und München war dies auch Stuttgart. Hier wurden die Arbeiten zügig vorangetrieben, weil nicht nur dem damaligen Leiter der Handschriftenabteilung, Wolfgang Irtenkauf, sondern auch Bibliotheksdirektor Hoffmann sehr viel an der Sache lag. Als vordringliches Projekt erschien die Erschließung der Codices der Hofbibliothek. Wiederum Johanne Autenrieth übernahm die Beschreibung des ersten Teils der so bezeichneten Codices ascetici (HB I 1-150), mit hauptsächlich monastischen, liturgischen und homiletischen Texten. Nach ihrem Weggang 1966 an die Universität Freiburg arbeiteten, quasi als Experten für diese Textgattung, der Beuroner Benediktiner Pater Virgil Fiala und Wolfgang Irtenkauf, ebenfalls Theologe, an diesem Katalog, der 1968 erschien ${ }^{12}$. 1970 bereits war der zweite Teil der Codices ascetici, von Fiala und Hermann Hauke verfass ${ }^{13}{ }^{3}$, gedruckt, $z$ wischenzeitlich auch ein Katalog mit etwas gemischten Gattungen, unter anderem Poetae Germanici, von Maria Sophia Buhl und Lotte Kurras ${ }^{14}$. In schneller Folge erschienen weitere vier Bände, die den restlichen Bestand der Hofbibliothek abdeckten, die letzten drei im Jahr 1975 ${ }^{15}$. Der gesamte Bestand der Hofbibliothek war damit innerhalb von nicht einmal 20 Jahren vollständig beschrieben und unter der Bezeichnung "Reihe 2" publiziert. Keine andere Bibliothek konnte eine vergleichbare Leistung vorweisen. Die Württembergische Landesbibliothek hat damit Pionierarbeit geleistet; nicht die einzige, wie im Weiteren noch darzustellen sein wird. Als weitere Frucht dieser Anstrengungen konnte Herrad Spilling den 1991 erschienenen Katalog der datierten Handschriften der Hofbibliothek erstellen ${ }^{16}$, immer noch einen der wenigen dieser Reihe bisher in Deutschland.

Parallel dazu erstellte der Musikwissenschaftler, Redakteur beim SDR, Komponist und Gründer der Schola cantorum Stuttgart, Clytus Gottwald, die ersten Kataloge in der Reihe der Musikhandschriften, auch dies eine Pionierarbeit. 1964 legte er einen ersten Band vor ${ }^{17}$, der unter anderem Werke der ehemaligen Hofkapelle des 16. Jahrhunderts erfasst, und schon 1965 den zweiten Band ${ }^{18}$, der die großen Chorbücher erschließt, die an die Hofbibliothek gegangen waren. Unter letzteren befinden sich die berühmten Lorcher Chorbücher. Zwei weitere Kataloge zu Musikhandschriften von Clytus Gottwald - im Wesentlichen Material der Hofoper - folgten 2000 und 2004. Für Musikalien gelten naturgemäß andere Beschreibungsgrundsätze als für Texthandschriften, zumal jene überwiegend neuzeitlich sind. Darauf kann hier nicht eingegangen werden ${ }^{19}$. Andere Regeln gelten auch für die in einer Sonderreihe erschienenen Kataloge von Ingeborg Krekler ${ }^{20}$.

Neben den Arbeiten an den Handschriften der Hofbibliothek stand als zweites Großprojekt die Erschließung der Bestände der Öffentlichen Bibliothek unter der Bezeichnung Reihe 1 an, für die es nur den un-zulänglichen alten Katalog in 13 Bänden gab. Pater Virgil Fiala und Wolfgang Irtenkauf begannen

(11) URL: http://www.manuscripta-mediaevalia.de/\#I4

(12) Johanne Autenrieth, Virgil E. Fiala, Wolfgang Irtenkauf, Die Handschriften der Württembergischen Landesbibliothek Stuttgart 2.1.1: HB I 1-150, Wiesbaden 1968.

(13) Virgil E. Fiala, Hermann Hauke, Die Handschriften der Württembergischen Landesbibliothek Stuttgart 2.1.2: HB I 151 - 249, Wiesbaden 1970.

(14) Maria Sophia Buhl, Lotte Kurras, Die Handschriften der Württembergischen Landesbibliothek Stuttgart 2.4.2: HB XI 1 - XIV 28, Wiesbaden 1969.

(15) Maria Sophia Buhl, Die Handschriften der Württembergischen Landesbibliothek Stuttgart 2.4.1: HB VIII 1 - X 30, Wiesbaden 1972; Helmut Boese, Die Handschriften der Württembergischen Landesbibliothek Stuttgart 2.2.1: HB /I 1 - IV 36, Wiesbaden 1975; Wolfgang Irtenkauf, Ingeborg Krekler, Die Handschriften der Württembergischen Landesbibliothek Stuttgart 2.2.2: HB V 1 - 105, Wiesbaden 1975; Magda Fischer, Die Handschriften der Württembergischen Landesbibliothek Stuttgart 2.5: HB XV 1 - HB XVI 1-28, Wiesbaden 1975.

(16) Herrad Spilling, Die datierten Handschriften der ehemaligen Hofbibliothek Stuttgart, Teil 1, bearb. auf Grund der Vorarbeiten von Wolfgang Irtenkauf, Stuttgart 1991 (Datierte Handschriften in Bibliotheken der Bundesrepublik Deutschland 3/1).

(17) Clytus Gottwald, Die Handschriften der Württembergischen Landesbibliothek Stuttgart, 1,1: Cod. Mus. Fol. I 1-71, Wiesbaden 1964.

(18) Clytus Gottwald, Die Handschriften der ehemaligen Hofbibliothek Stuttgart, 6.1. HB XVII 1-28, Wiesbaden 1965.

(19) Richtlinien, wie Anm. 10, S. 51-62.

(20) Ingeborg Krekler, Katalog der handschriftlichen Theaterbücher des ehemaligen Württembergischen Hoftheaters (Codices theatrales), Wiesbaden 1979: Ingeborg Krekler, Die Autographensammlung des Stuttgarter Konsistorialdirektors Friedrich Wilhelm Frommann (17071787), Wiesbaden 1992. 


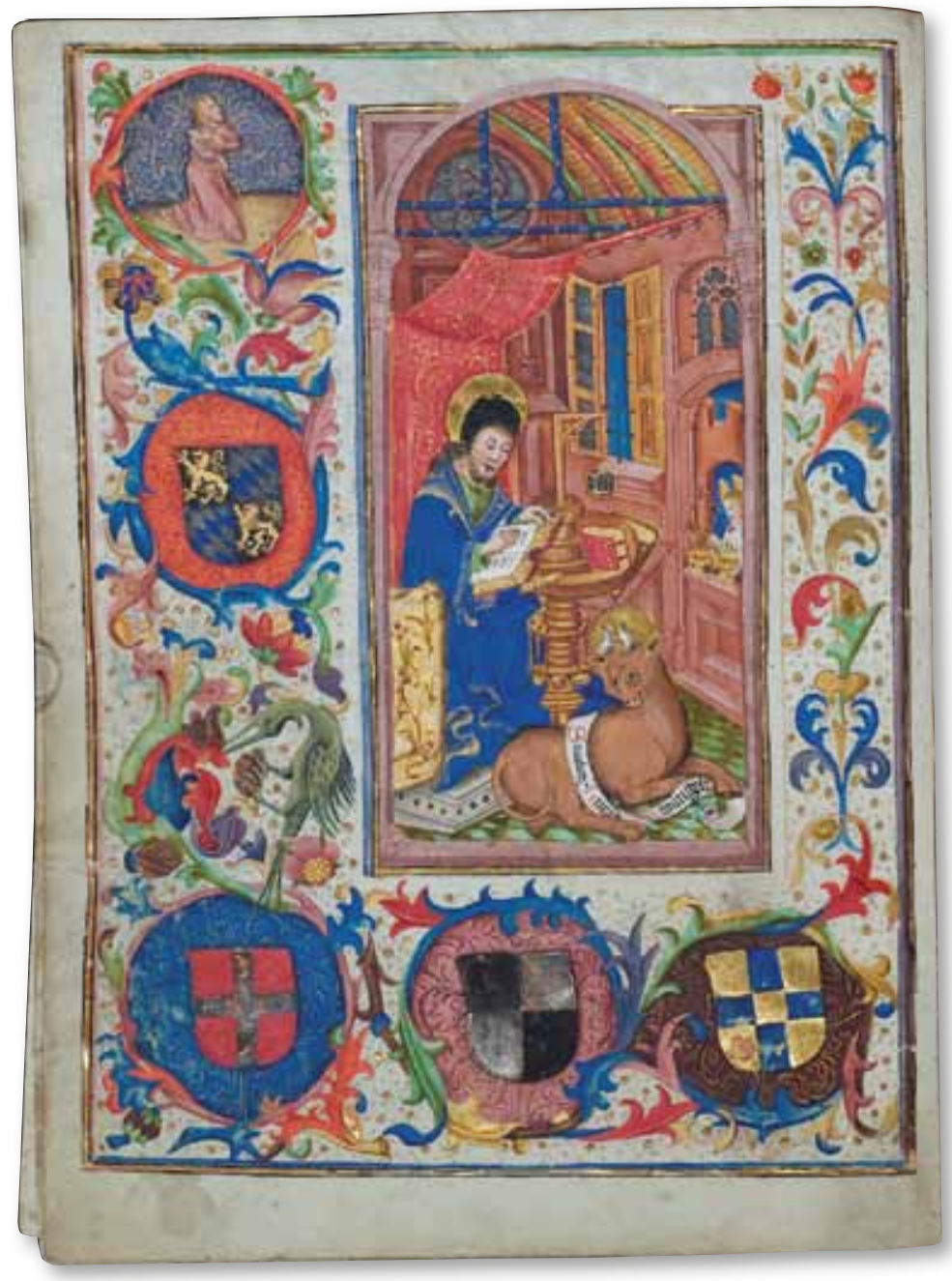

Abb. 2: Cod. brev. 1, BI. 2v

1969 mit den Codices breviarii, Fiala erkrankte aber 1974 schwer, und Irtenkauf führte die Arbeiten zu Ende, so dass der Katalog 1977 erscheinen konnte ${ }^{21}$. Als vorläufig letzten Band der Reihe 1 gaben 1981 Irtenkauf und Ingeborg Krekler die Beschreibungen der Codices poetici heraus ${ }^{22}$. Überwiegend finden sich hier schwäbische Größen wie u.a. Hölderlin, Schiller und Mörike, daneben aber auch Werke wie

(21) Virgil E. Fiala, Wolfgang Irtenkauf, Die Handschriften der Württembergischen Landesbibliothek Stuttgart 1.3: Codices breviarii, Wiesbaden 1977.

(22) Wolfgang Irtenkauf, Ingeborg Krekler, Die Handschriften der Württembergischen Landesbibliothek Stuttgart 1.2: Codices poetici et philologici, Wiesbaden 1981

(23) Sven Limbeck, Wolfgang Metzger, Die Handschriften der Württembergischen Landesbibliothek Stuttgart 1.5: Codices biblici in quarto et in octavo: (Cod. bibl. $4^{\circ} 1-46$ und cod. bibl. $\left.8^{\circ} 1-20\right)$, Wiesbaden 2013.

(24) Karl August Barack, Die Handschriften der Fürstlich-Fürstenbergischen Hofbibliothek zu Donaueschingen, Tübingen 1865.

(25) Regina Hausmann, Die theologischen Handschriften der Hessischen Landesbibliothek Fulda bis zum Jahr 1600. Codices Bonifatiani 1-3, Aa 1-145a. - Wiesbaden: Harrassowitz, 1992. - (Die Handschriften der Hessischen Landesbibliothek Fulda; Bd. 1); Regina Hausmann, Die Historischen, Philologischen und Juristischen Handschriften der Hessischen Landesbibliothek Fulda bis zum Jahr 1600. B 1 - 25, C 1-18.68, D1 - 48. Beschrieben von Regina Hausmann. Wiesbaden: Harrassowitz, 2000. (Die Handschriften der Hessischen Landesbibliothek Fulda, Bd. 2).

(26) Ausführliche Darstellung bei: Peter Burkhart, in: Das Mittelalter. Perspektiven mediävistischer Forschung. Zeitschrift des Mediävistenverbandes 14/2009, S. 110-124. ein griechisch-lateinischer Homer, ein Roman de la rose und Werke antiker Autoren aus dem Besitz deutscher Humanisten. Erst 2013 erschien Band 5 der Reihe, der Katalog der Codices biblici im Quart- und Oktavformat ${ }^{23}$, begonnen von Sven Limbeck und - nach dessen Fortgang nach Wolfenbüttel - abgeschlossen von Wolfgang Metzger. Der von Regina Hausmann erstellte und von Carsten Kottmann auf den neuesten Stand gebrachte Katalog der Codices biblici in Folio wird demnächst erscheinen, ebenso Sven Limbecks Bearbeitung derjenigen Donaueschinger Codices in Stuttgart, die im Katalog von Barack ${ }^{24}$ nicht erfasst wurden. Derzeit laufende Projekte sind die Katalogisierung der mittelalterlichen Codices theologici in Folio durch Wolfgang Metzger und der neuzeitlichen Codices theologici in Folio durch Carsten Kottmann.

Wie oben schon erwähnt, besteht die Funktion der Handschriftenzentren auch darin, die Katalogisierung der Bestände anderer Bibliotheken zu übernehmen und zu betreuen. In Stuttgart bearbeitete Regina Hausmann die Handschriftenbestände der Hessischen Landesbibliothek Fulda, die in großer Zahl aus dem oberschwäbischen Kloster Weingarten stammen. Dieses Kloster stellt für die Sammlung der Württembergischen Landesbibliothek die bedeutendste Provenienz dar. Daraus ergaben sich vor Ort hervorragende Vergleichsmöglichkeiten, und generell kommt die Anwesenheit mehrerer Bearbeiter an einem Ort wegen der Möglichkeiten des direkten Austauschs mit den Kollegen der Qualität der Arbeit zugute. Die beiden Fuldaer Kataloge erschienen 1992 und $2000^{25}$. Seit einigen Jahren leistet Ulrike Spyra die entsagungsvolle Arbeit der Katalogisierung der mittelalterlichen Handschriften der Universitätsund Landesbibliothek Darmstadt im Handschriftenzentrum, deren erstes Teilprojekt in diesem Jahr seinen Abschluss finden wird.

\section{Die Katalogisierung der illuminierten Handschriften ${ }^{26}$}

Zuletzt ist noch auf die illuminierten Handschriften einzugehen, die den attraktivsten Teil jeder Sammlung darstellen. In der dritten Auflage der Richtlinien 
1983 waren erstmals auch Regeln für die Handschriftenbeschreibung unter kunsthistorischem Aspekt ${ }^{27}$ aufgenommen worden. Es handelt sich dabei um dieselben Codices, die auch in den allgemeinen Katalogen als sogenannte Texthandschriften beschrieben werden, soweit sie einigermaßen bemerkenswerten Buchschmuck enthalten. Darauf liegt nun das Gewicht des Katalogisats, die Texte werden dagegen nur knapp benannt. Allerdings ist gerade bei figürlichen Darstellungen der Textbezug herzustellen, in Stundenbüchern zum Beispiel das betreffende Offizium zu nennen oder in epischen Dichtungen die Verse, auf die sich das jeweilige Bild bezieht. Die Darstellung des Äußeren und der Geschichte ist grundsätzlich die gleiche, nur in etwas anderer Anordnung. Zweiter zentraler Teil des Katalogisats nach der beschreibenden Darstellung der einzelnen Elemente des Buchschmucks ist der Abschnitt "Stil und Einordnung", in dem die Argumente für Lokalisierung, Datierung, kunsthistorische und eventuell geistesgeschichtliche Einordnungen dargelegt werden. Während aber die allgemeinen Kataloge Beschreibungen nach Signaturengruppen und darin nach laufenden Nummern bieten, werden hier Handschriften gemäß Entstehungszeit und Entstehungsregion ohne Rücksicht auf die Signatur zusammengestellt. Zusätzliche Register zu Buchschmuck sowie den zum Vergleich herangezogenen Handschriften und anderen Kunstwerken ermöglichen den Zugang unter spezifisch kunsthistorischen Aspekten; dazu erleichtert eine Konkordanz der Signaturen und Katalogisat-Nummern das Auffinden der Einzelbeschreibungen. Kataloge illuminierter Handschriften unter der Ägide der DFG existieren nur für die größeren Bibliotheken mit genügend großen Beständen, die den Aufwand der Erforschung und des Drucks lohnen, bisher nur für München, Bamberg, Berlin und Stuttgart.

\section{Gliederungen und Grundsätze der Beschreibung} mussten aber erst erarbeitet werden. Das Projekt zur Erschließung der einschlägigen Bestände wurde von Wilhelm Hoffmann und Wolfgang Irtenkauf ebenfalls schon um 1960 ins Auge gefasst, und so befand sich die Württembergische Landesbibliothek auch hier in einer Pionierrolle. Mit den Vorarbeiten, der Sichtung der Bestände und ihrer vorläufigen Einordnung nach kunsthistorischen Gesichtspunkten wurde Sigrid von Borries-Schulten betraut. Dem Gesamtprojekt legte man zunächst eine grobe Eintei- lung nach der gängigen Epochengliederung karolingisch, ottonisch, romanisch und gotisch zugrunde. Weil aus den beiden ersten Epochen vergleichsweise wenige Codices in Frage kamen, fasste man sie zur vorromanischen Zeit zusammen. Man begann aber nicht mit diesem ältesten Bestand, sondern mit den romanischen Handschriften aus Zwiefalten. Die Bibliothek dieses Klosters war mit der Säkularisation praktisch vollständig nach Stuttgart gekommen. Das Zwiefaltener Skriptorium hatte speziell im 12. Jahrhundert auch in künstlerischer Hinsicht den Höhepunkt seiner Produktion erreicht. Da von den 84 zu bearbeitenden Codices 73 in Zwiefalten selbst entstanden waren, bot sich die Möglichkeit, einen nahezu geschlossenen Bestand präsentieren zu können und erstmals eine kunsthistorische Studie über ein klösterliches Skriptorium in Südwestdeutschland vorzulegen.

Über einige Jahre konnte man sich nicht entscheiden, wie die Anlage der Katalogisate aussehen, wie das Verhältnis der einzelnen Aspekte der Beschreibung zum eigentlichen kunsthistorischen Teil sein sollte und wie detailliert die Darstellung von Ornamentik und figürlichem Schmuck sein sollte; Richtlinien dazu erschienen ja erstmals $1983^{28}$. Zum Durchbruch verhalf der erste Münchener Katalog illuminierter Handschriften von Elisabeth Klemm ${ }^{29}$, der zur Grundlage für die 1983 herausgegebenen Richtlinien wurde. Der wichtigste Stuttgarter Beitrag zur bis heute gültigen Form bestand in der Hinzufügung des Abschnitts "Stil und Einordnung". 1987 erschien der Katalog von Borries-Schulten ${ }^{30}$, gleichzeitig der andere Katalog der romanischen Handschriften in Stuttgart ${ }^{31}$, der die weiteren Provenienzen behandelt, unter denen Weingarten den größten Anteil hat. Auch unter Hoffmanns Nachfolger Hans-Peter Geh (seit 1970) und dem Leiter der Handschriftenabteilung, Felix Heinzer, verfolgte man kontinuierlich die Erschließung der Sammlung. Den Katalog zu den Handschriften des 13. Jahrhun-

(27) Richtlinien, wie Anm. 10, S. 29-33.

(28) S. Anm. 27.

(29) Elisabeth Klemm, Katalog der illuminierten Handschriften der Bayerischen Staatsbibliothek in München 3.1. Die romanischen Handschriften der Bayerischen Staatsbibliothek. Teil 1: Die Bistümer Regensburg, Passau, Salzburg. Text- u. Tafelbd., Wiesbaden 1980.

(30) Sigrid von Borries-Schulten, Die illuminierten Handschriften der Württembergischen Landesbibliothek Stuttgart 2: Die romanischen Handschriften der Württembergischen Landesbibliothek Stuttgart, Bd. 1: Provenienz Zwiefalten (Denkmäler der Buchkunst 7), Stuttgart 1987.

(31) Annegret Butz, Katalog der illuminierten Handschriften der Württembergischen Landesbibliothek Stuttgart 2. Die romanischen Handschriften der Württembergischen Landesbibliothek Stuttgart, Bd. 2: Verschiedene Provenienzen (Denkmäler der Buchkunst 8), Stuttgart 1987. 
derts $^{32}$ erarbeitete Christine Sauer. Zunächst hatte Ulrich Kuder damit begonnen, der aber bald auf eine Professur nach Kiel wechselte. Hier präsentiert sich ein deutlich anders gearteter Bestand als in den Vorgängerbänden. Neben Südwestdeutschland als Haupteinzugsgebiet der Landesbibliothek sticht die große Zahl (49) der Codices aus Frankreich, darunter die meisten aus Paris, hervor, die durch die dominierende Rolle der Pariser Universität in den Wissenschaften der Zeit, vor allem der Theologie, erklärbar ist. Seit dem 12. Jahrhundert hatte sich, von Paris ausgehend, das Fleuronné als neue Art des Initialschmucks in vielfältigen Erscheinungsformen entwickelt, für die es aber nur eine sehr eingeschränkte Terminologie gab. Christine Sauers Katalog ist deshalb unter anderem auch ein Beitrag zu deren Erweiterung und Präzisierung, besonders was Süd-

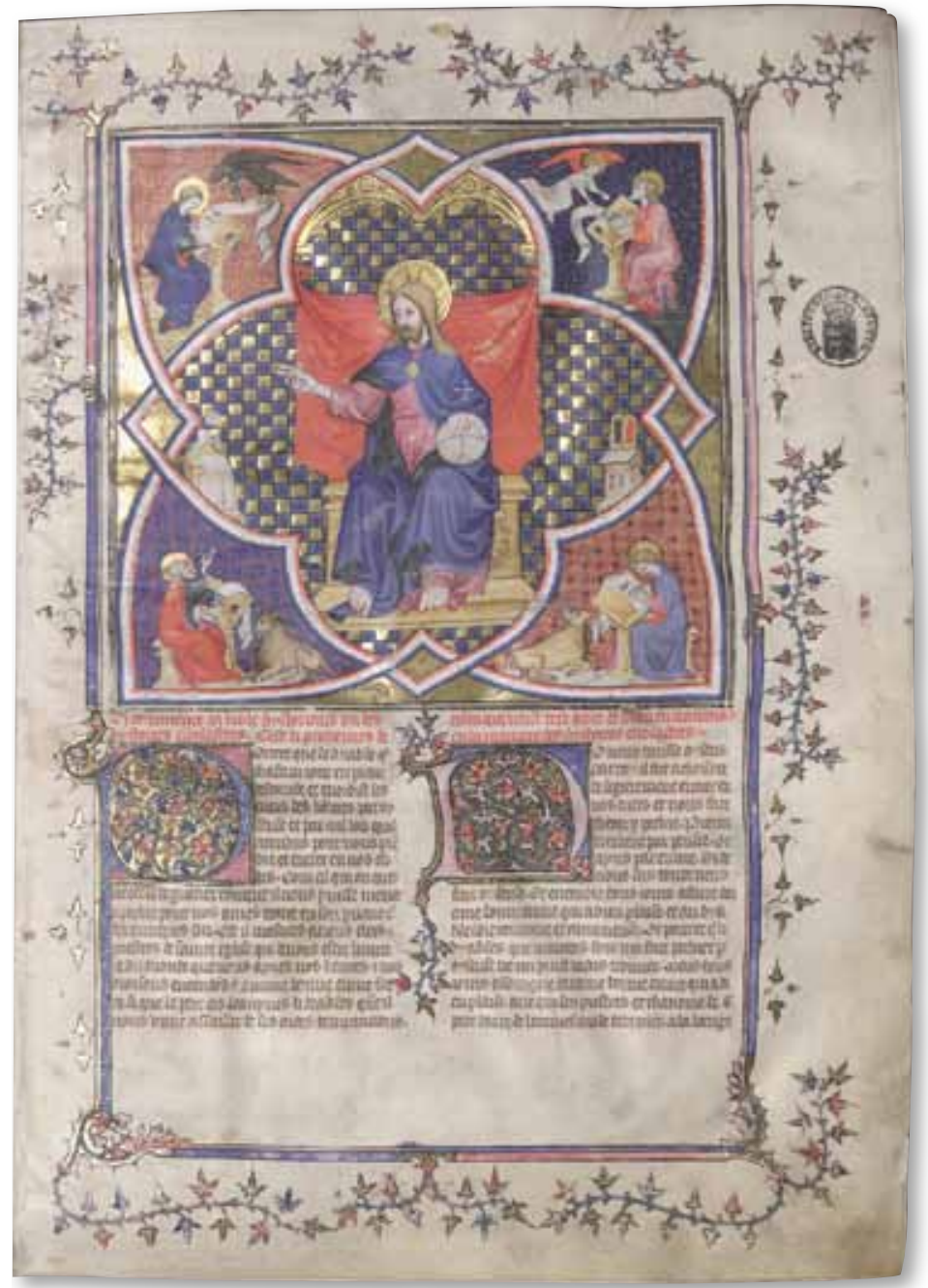

Abb. 3: Cod. bibl.fol. 6, BL. 3r

(32) Christine Sauer, Katalog der illuminierten Handschriften der Württembergischen Landesbibliothek Stuttgart 3. Die gotischen Handschriften der Württembergischen Landesbibliothek Stuttgart, Teil 1: Vom späten 12. bis zum frühen 14. Jahrhundert (Denkmäler der Buchkunst 12), Stuttgart 1996.

(33) Peter Burkhart, Die illuminierten Handschriften der Württembergischen Landesbibliothek Stuttgart 3: Die gotischen Handschriften der Württembergischen Landesbibliothek Stuttgart, Band 2: Vom späten 13. bis zum frühen 15. Jahrhundert, Wiesbaden 2005.

(34) Peter Burkhart, in: Scriptorium 53/1999, S. 187-199. westdeutschland betrifft. Frau Sauer nahm danach die Fortführung des Projekts mit den Handschriften des 14. Jahrhunderts in Angriff, wechselte aber nach einigen Monaten nach Nürnberg. Die Bearbeitung wurde nach kurzer Vakanz vom Autor dieses Berichts übernommen; der Katalog erschien $2005^{33}$. Wieder wandelt sich der Charakter des Bestands. Umfangreiche Dichtungen in deutscher Sprache treten als neue Gattung auf, ebenso Stundenbücher für die private Andacht. Im Zuge der Arbeit konnte die Zugehörigkeit einer Bible historiale (Cod. bibl. $2^{\circ} 6$ ) zur Bibliothek des Louvre nachgewiesen werden ${ }^{34}$.

Der lange zurückgestellte Wunsch, endlich die ältesten Schätze der Bibliothek zu erschließen, sollte nun befriedigt werden. Die sogenannten vorromanischen Codices verteilen sich über einen vergleichsweise langen Zeitraum von ca. 730 bis in die zweite Hälfte des 11. Jahrhunderts. Es befinden sich zwar keine Exemplare der berühmten Hofschule Karls des Großen oder der Reichenauer Schule des 10. Jahrhunderts darunter, aber neben dem in einem Stil aus insularen und merowingischen Elementen geschmückten Psalter aus Echternach (Cod. bibl. $2^{\circ} 12 \mathrm{a}-$ c) und zwei Evangeliaren der Kölner ottonischen Schule (Cod. bibl. $4^{\circ}$ 2a-b; Cod. bibl. $2^{\circ}$ 21 ) ragt durch seinen umfangreichen und im Stil einzigartigen Bildschmuck der Stuttgarter Psalter (Cod. bibl. $2^{\circ} 23$ ) heraus. Im Übrigen dominieren Codices aus dem erweiterten Bodenseeraum mit den Zentren Reichenau und Sankt Gallen. Der Druck des Katalogs wird dieses Jahr erfolgen.

Auch Bibliotheksdirektor Hannsjörg Kowark und die neue Leiterin der Handschriftenabteilung, Kerstin Losert, ließen keine Unterbrechung der Arbeiten eintreten. Es fehlten noch die Bestände des 15. und des frühen 16. Jahrhunderts, die wegen der Menge des Materials auf zwei Projekte verteilt werden mussten mit dem Schnitt bei etwa 1475. Im Gegensatz zur Handhabung beim Erfassen der ältesten Handschriften konnten hier einfachere Schmuckformen nicht mehr berücksichtigt werden. Die Werke des ersten Teilprojekts entstammen zu vier Fünfteln deutschsprachigen Gebieten mit dem Schwerpunkt Südwestdeutschland, wozu auch Elsass und Nordschweiz gerechnet 
werden. Besonders vertreten sind Werke deutscher Sprache mit umfangreichen Illustrationszyklen aus kolorierten Federzeichnungen, vor allem naturkundliche Werke (Konrad von Megenberg: Cod. med. et phys. $2^{\circ} 14$ ) und epische Dichtungen (Rudolf von Ems: Wilhelm von Orlens). Die Werkstatt Diebold Laubers in Hagenau war ein Zentrum der Produktion solcher Bücher. Bemerkenswert ist auch eine Gruppe von Handschriften, anhand derer die Entwicklung der Augsburger Buchmalerei gezeigt werden kann, die in dieser Zeit eine führende Stellung einnahm.

Weil die Zeit bis zum Ruhestand des bisherigen Bearbeiters Peter Burkhart nicht mehr gereicht hätte, um die Katalogisierung des noch ausstehenden Bestands abzuschließen, wurde Wolfgang Metzger mit einem Teil der Aufgabe betraut. Ihm fiel dabei die $24 \quad$ Erschließung der Handschriften ab etwa 1500 bis ca. $1530 \mathrm{zu}$, soweit sie noch stilistisch und inhaltlich dem Mittelalter zuzurechnen waren. In dieser inhaltlich gemischten Gruppe sind mit mehreren Stücken unter anderem deutsche Stunden- und Gebetbücher vertreten; am bekanntesten und für Südwestdeutschland bedeutend sind jedoch die drei Lorcher Chorbücher (Cod. mus. I $2^{\circ}$ 63-65). Im ersten Teil bildet neben einigen italienischen Prachthandschriften eine größere Anzahl von französischen und flämischen Stundenbüchern zum Teil hoher Qualität eine bemerkenswerte Gruppe. Als Zeugnis für den südwestdeutschen Humanismus, speziell in Heidelberg, können etliche importierte italienische Handschriften zusammen mit pfälzischen Nachahmungen solcher Werke gelten. Damit ist die Katalogisierung des gesamten Bestandes an illuminierten Codices der Württembergischen Landesbibliothek abgeschlossen. Ein Großteil der Beschreibungen ist durch Maria Gramlich und Hauke Hillebrecht bereits in die Handschriftendatenbank eingegeben. Damit bleibt nur noch die Drucklegung der beiden Bände zum 15. Jahrhundert vorzubereiten.

Peter Burkhart 